\title{
Endogenous Thrombin Potential Area Under Curve Measurement
}

National Cancer Institute

\section{Source}

National Cancer Institute. Endogenous Thrombin Potential Area Under Curve

Measurement. NCI Thesaurus. Code C102263.

The measurement of the area under the thrombin generation curve. 